\title{
THE USE OF MULTIPLE DATA SOURCES IN THE PROCESS OF TOPOGRAPHIC MAPS UPDATING
}

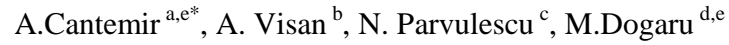 \\ ${ }^{a}$ Dep. Of Cartography and Photogrammetry, National Centre of Cartography, Bucharest, Romania - adriana.cantemir@cngcft.ro \\ ${ }^{\mathrm{b}}$ Dep. Of Cartography and Photogrammetry, National Centre of Cartography, Bucharest, Romania - alexandru.visan@cngcft.ro \\ ${ }^{c}$ Dep. Of Cartography and Photogrammetry, National Centre of Cartography, Bucharest, Romania - nicoleta.parvulescu@cngcft.ro \\ ${ }^{\mathrm{d}}$ Dep. Of Cartography and Photogrammetry, National Centre of Cartography, Bucharest, Romania - margarita.dogaru@ancpi.ro \\ ${ }^{\mathrm{e}}$ Romanian Society of Photogrammetry and Remote Sensing, Bucharest, Romania
}

Commission IV, WG IV/1

KEY WORDS: LiDAR, DTM, Hydrohraphy, Satellite images

\begin{abstract}
:
The methods used in the process of updating maps have evolved and become more complex, especially upon the development of the digital technology. At the same time, the development of technology has led to an abundance of available data that can be used in the updating process. The data sources came in a great variety of forms and formats from different acquisition sensors. Satellite images provided by certain satellite missions are now available on space agencies portals. Images stored in archives of satellite missions such us Sentinel, Landsat and other can be downloaded free of charge. The main advantages are represented by the large coverage area and rather good spatial resolution that enables the use of these images for the map updating at an appropriate scale. In our study we focused our research of these images on 1: 50.000 scale map. DEM that are globally available could represent an appropriate input for watershed delineation and stream network generation, that can be used as support for hydrography thematic layer update. If, in addition to remote sensing aerial photogrametry and LiDAR data are ussed, the accuracy of data sources is enhanced. Ortophotoimages and Digital Terrain Models are the main products that can be used for feature extraction and update. On the other side, the use of georeferenced analogical basemaps represent a significant addition to the process. Concerning the thematic maps, the classic representation of the terrain by contour lines derived from DTM, remains the best method of surfacing the earth on a map, nevertheless the correlation with other layers such as Hidrography are mandatory. In the context of the current national coverage of the Digital Terrain Model, one of the main concerns of the National Center of Cartography, through the Cartography and Photogrammetry Department, is represented by the exploitation of the available data in order to update the layers of the Topographic Reference Map 1:5000, known as TOPRO5 and at the same time, through the generalization and additional data sources of the Romanian 1:50 000 scale map. This paper also investigates the general perspective of DTM automatic use derived products in the process of updating the topographic maps.
\end{abstract}

\section{INTRODUCTION}

\subsection{Motivation}

Since their emergence, topographic maps have had, as a main purpose, the achievement of the best terrain representation by conventional means of the planimetric details and the 3D topographic surface. For this task mathematical models have been used to ensure the cartographic representation, associated with a $\mathrm{X}, \mathrm{Y}, \mathrm{Z}$ reference coordinate system describing the position of the objects from reality.

It is known that every representation is valid at the moment of its realization, since the real world is in a continuous change, more or less dynamic. For this reason the process of updating the topographic maps is a permanent necessity. Even if is performed following a regular scheduler, or at a convenient time, the moment of the update it's consider the forth dimension for a topographic map after X, Y, Z, coordinates.
The methods used in the process of updating a map have evolved and become more divers, especially upon the development of the digital technology. In order to achieve the desired precision in an efficient manner, different methods are adopted for the update of the small surface areas, where total station or GNSS measurement are used, or for the update of large areas, where photometric methods for collecting date are more suitable, even if the platform is a satellite, an airplane, a helicopter, or a Unmanned Aerial Vehicle.

Photogrametry offers a great deal of possibilities and method combination for obtaining a digital map. In the case of Hydrographic Network the best method for collecting or updating the course of the rivers is stereo restitution on absolute orientated models. Being aware of the correlation between the topographic surface and the Hydrographic the use of the stereo model ensures the caption of the planimetric position(X, Y), along with the height information, $\mathrm{Z}$. The main inconvenient of the stereo restitution method is represented by the high cost of the process due to expensive equipments, highly qualified personnel and time consuming exploitation methods.

\footnotetext{
* Adriana Cantemir
} 


\subsection{Methods}

In recent years, from the quality - costs perspective for making and updating the topographic plans, the most popular methods had become the photo interpretation from the ortho photo images. The results obtained from this method have their limitations according to the "plan" characteristic of this photogrammetric product (2D information) and of its specific quality, evaluated by values of the precision and resolution or by functions representing the quality of the digital image. Among these limitations, the lack of height information for the planimetric results can not be overcame. But.....it can be provided by other means. A data source that supplies the $3 \mathrm{D}$ information with a satisfactory accuracy is represented by the Digital Terrain Model (DTM) or the Digital Surface Model (DSM), obtained out of the scans made by LiDAR devices.

We refer to the accuracy as both the precision on the 3 coordinates $\mathrm{X}, \mathrm{Y}, \mathrm{Z}$ and the issues such as: resolution (ortho photo images or DTM/DSM), image quality, the quality of point classification, the quantity of information that can be extracted (from the ortho photo images, DTM), topicality of the data (the moment they were created). To photo interpret these two kinds of data correctly it is recommended that they have the same accuracy.

Amongst the miscellaneous themes of the topographic plan, the Hydrographic one is one of the most difficult. This work is showing some aspects and a workflow for updating the Hydrographic theme of the Romanian Topographic Plan according to the 1:5.000 scale, named TopRo5. Achieving and updating this digital map is a law stipulated objective for the National Center of Cartography.

The updating is being done for the elements with a polyline geometry type representing the axis of the running waters and accordingly a polygon geometry type for the still waters. The data model is a default one and it meets the INSPIRE Directive for this theme.

The updating procedure that we are about to present is a response to the needs of the actual referenced topographic map, of the evolution and diversity of the data source that can be used to obtain or update the Hydrographic representation for the Topographic Maps as it may also represent a freestanding theme. It is also a response for the needs of updating other themes that are directly influenced by the Hydrographic representation as a support, such as "relief" or the use of geospatial data from the TopRo5 project for other derived plans an maps, such as the digital Romanian Map for scale 1: 50.000. Furthermore, the existence of some versions for these themes are according to the requirements for an unique infrastructure of spatial data in Europe.

This case study is a pleading for the importance that must be given to the correlation of the Topographic plans and maps themes and also of its influence on one another or the interdependence of the Hydrographic and Relief Theme.

\section{DATA SOURCES}

\subsection{Current status}

The updating methods for any cartographic support requires data sources that need to respond to several criteria such as: precision, complexity and topicality. But the achievement of such data set according to these principles supposes important resources for their collection and processing.
The photogrammetric methods offer a good enough alternative for the updating process. The Stereo restitution method, based on a stereoscopic model represents the classic and certain process, and also laboriously and time consuming.

In the case of updating the Hydrographic Network, an efficient method of data exploitation might be the use of a data combination made out of ortho photo images and DTM obtained on the basis of LiDAR data collection. Thus, a vectorization of the hydrographic network may be performed, or it could be generated automatically. Satellite imagery represents an other data source that allowes to better understand the specific themes.

The theme that is about to be updated is the result of the $2 \mathrm{D}$ photo interpretation on ortho photo images that were made after the sequentially flights taken since 2005 to 2013 . That means that the accuracy differs between the years.

This issue is the same for all the other themes within the TopRo5 Project. Obviously, all the other plans and maps are in the same situation.

In this case the need to update both the Hydrographic Theme and the Relief Theme, represented by contour lines is obviouse, dedicating at the same time a substantial attention to their interaction (Heidemann, 2014).

The 2D photo interpretation does not ensure the geometric accuracy of the obtained vectors because they are drawn by interpretation of the different colors from the ortho photo images and having no information on the height coordinate. These height coordinates that are gathered together in a digital model representing the third coordinate offers the relief aspect and slopes and ensures the essential condition for water flow.

\subsection{Sources of error}

It may be concluded that any photo interpretation of the Hydrographic network in a 2D environment cannot supply a certain or at least satisfactory result for the referenced plan. This statement can be exemplified by some frequently met types of errors in the $2 \mathrm{D}$ environment.

A coarse error may happen when in hydro technical, a channel (without slope) is interpreted as a channeled running water (with slope). In these situations, where the dimensions and chromatic aspects are alike, when there is lack of information regarding the slope, a certain difference between a channel and a running water is difficult to make.

Another frequent mistake occurs in the flat areas covered with forests, where narrow roads with irregular shape look like running waters.

The course of the running waters and their slopes may be easily be misinterpreted.

The track of the dry rivers or with a variable flow and the excess moisture in the fields cannot properly be estimated in the case of the lack of relief.

In the areas covered with thick forests and the aerial images taken in a period with developed vegetation, the track of running waters is difficult to spot, even impossible. The tracks of the running waters that cross the residential areas, covered with buildings and vegetation are also barely visible without the 3D information. 

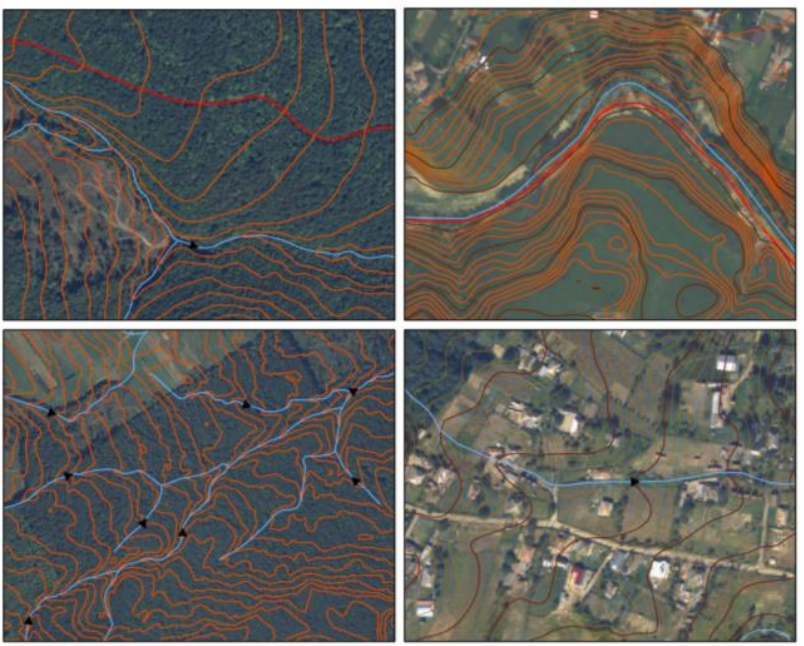

Figure 1. Photo interpretation from 2D and 3D data sources

Furthermore, the running water vectors are only $\mathrm{X}, \mathrm{Y}$ and when their track does not meet the relief, any further transformation to $3 \mathrm{D}$ space system is affected by errors.

The final errors for Hydrographic Network obtained by a 2D vectorization have random or systematic causes. A part of the systematic errors are included in the procces of producing the ortho photo images, used as main source of date for data colection. Besides the images resolution and radiometric quality, the ortho photo plan gathers all the positioning errors prior its processing (geodetic measurements, aerial triangulation, DTM generation, ortho rectification, the mozaicing of the ortho rectified images.)

\subsection{Proposed approach}

For diminishing the effects of the hydrographic errors obtained in a $2 \mathrm{D}$ environment, the use of height related information becomes mandatory. The data used should have the same accuracy or even better than 2D data. A possibility may be the use of a DTM generated in the ortho photo production process. For lower precisions this solution may be applied if the DTM was revised for at least the major errors.

For higher precision, a suitable way may be to use the DTM obtained by LiDAR scanning (NOOA, 2012). The LiDAR data ensure a good accuracy and they can be obtained separately from any other photogrammetric products and in a relatively short time, offering an important support in the process of hydrographic and relief updating.

The coverage with LiDAR data on an national level is in continuous growth, becoming an important data source. Considering that the collection of this kind of information is rather new in Romania, its quality, in general is not yet homogeneous, but it's improving.

Is a well known fact that the updating proces is conditioned by several factors, from which the data type and accuracy of the data dictates the method used in the precess and the expected results.

For Hydrographic Network updating or vector collection the LiDAR data can be considered an appropriate data source that must be complete, accurate and homogeneous. When transformed into proper formats, the LiDAR data may provide the basis for the automatic generation of the water flows. Unfortunately, the data is not always of the best quality and then we must use other sources of information. These are: ortho photo plans, different other georeferenced analog maps and plans and even measurement from the field.

\section{AUTOMATIC GENERATION OF WATER FLOW}

\subsection{Procedure steps}

The most efficient way of obtaining the hydrographic network by using LiDAR data should be the use of automatic methods. In our study we investigated the use of available LiDAR data for generating the water courses. Thus, there can be used specific methods of hydrodynamic modeling in ArcMAP tools, respectively the Hydrology Tool (ArcGIS Resources, 2016). Same kind of tools may also be found in other programs.

The operations are using raster data and they follow up the next steps:

- The identification and the discharge of some surface failings, such as data gaps or data out of pattern (spike, sink) for obtaining a valid terrain model for further processing (Greenlee, 1987);

- The creation of a raster with the flowing direction for every pixel;

- On the basis of the flow direction another raster will be made with the same dimensions with the pixels value equal with the figure that represents the number of the summoned flows towards it. This raster represents the accumulations of all the licks in every point;

- The raster classification on the basis of the representative value that differentiate the pixels on the water track. This value is known as "threshold". By classification we have 2 classes of pixels: water and non-water;

- The last step involves transforming the pixels clasified as „water" into vectors.

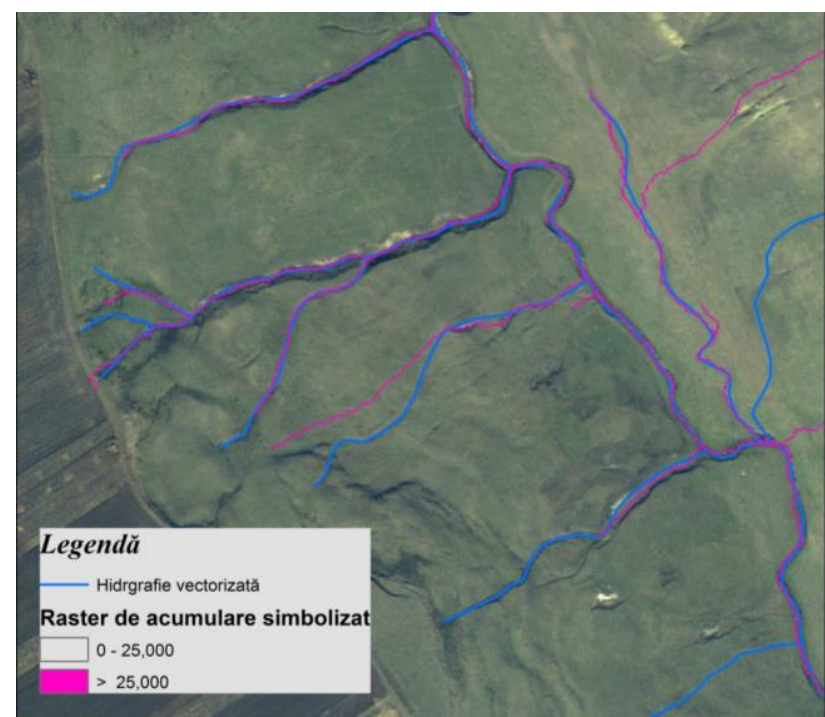

Figure 2. Comparison between the water courses generated by LiDAR data and the courses verctorized on the ortho photo

For the example presented above the raw Lidar data had a mean spatial resolution of $0.5 \mathrm{~m}$. In this case the results are optimal as a primary or secondary data source for the updating of the 1:5000 scale map. 
Different conclusion are to be made for the 30m SRTM global available model. We considered using the above procedure to delineate the hydrography theme as a line feature class for 1:50.000 scale map. Even if there are some areas where the results are satisfactory in most of the cases the path of the watercourse does not mach the reality in the field.

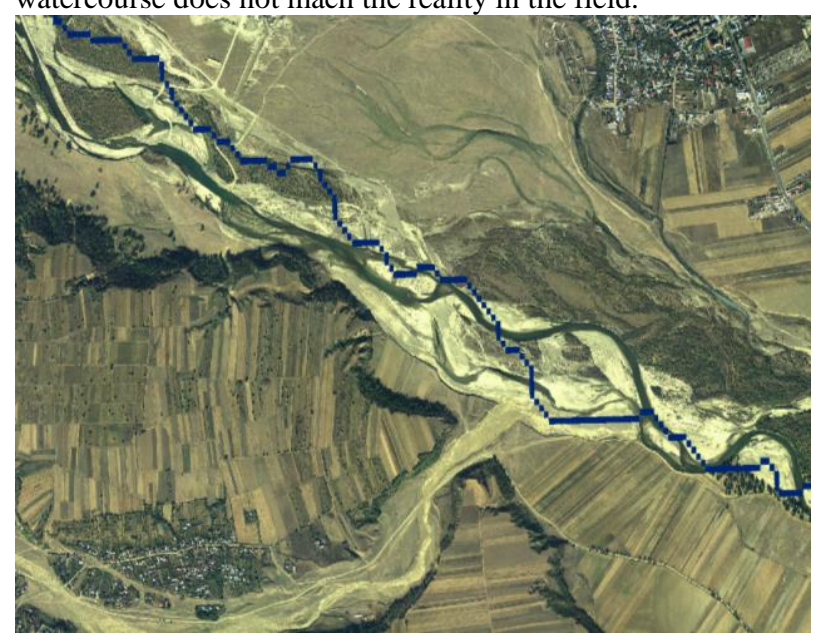

Figure 3. Obvious differences between the real path of the watercourse and the $30 \mathrm{~m}$ SRTM delineated course

\subsection{Remarks on the workflow}

The entire process uses the flowing model D8 and the approach proposed by Jenson and Domingue (1988).

The watercourse delineation method offers correct result if the area of an entire watershed is subjected to the analysis. The value of the threshold dictates the degree of consistency of the watercourses generated over the entire area.

The precision for obtaining the hydrographic network route depends on the default parameters and on the LiDAR data accuracy and spatial resolution. With some properly data, good results can be obtained in order to optimize a proper updating.The level of water track detail depends on the initial resolution of the height data. The better the resolution, the more detailed the track will be.To generate the flowing directions raster we must take into account the DTM precision. For example for the initial datawith $\mathrm{a}+/-15 \mathrm{~cm}$ precision, the points heights must have only 2 significant figures after comma (centimeters), or on the contrary the flowing directions could be wrong, taken into account too smaller height differences that may not always be real.

The pixels values that create the accumulation raster are directly influenced by the pixels position on the running water.

A pixel situated uphill will have a smaller accumulation value than one situated downhill. Also, a pixel situated at the confluence of two or more running waters will have a higher value.These observations must be taken into account when choosing the classification threshold value for the accumulation raster.The threshold value for classification has influence on the obtained results, especially on the generated water courses length and also on the water density for the entire map. A great value for the classification threshold results in shorter waters and a small value in longer waters.

The same way goes to running waters density (the short ones will be missing in the case of greater values for the threshold).

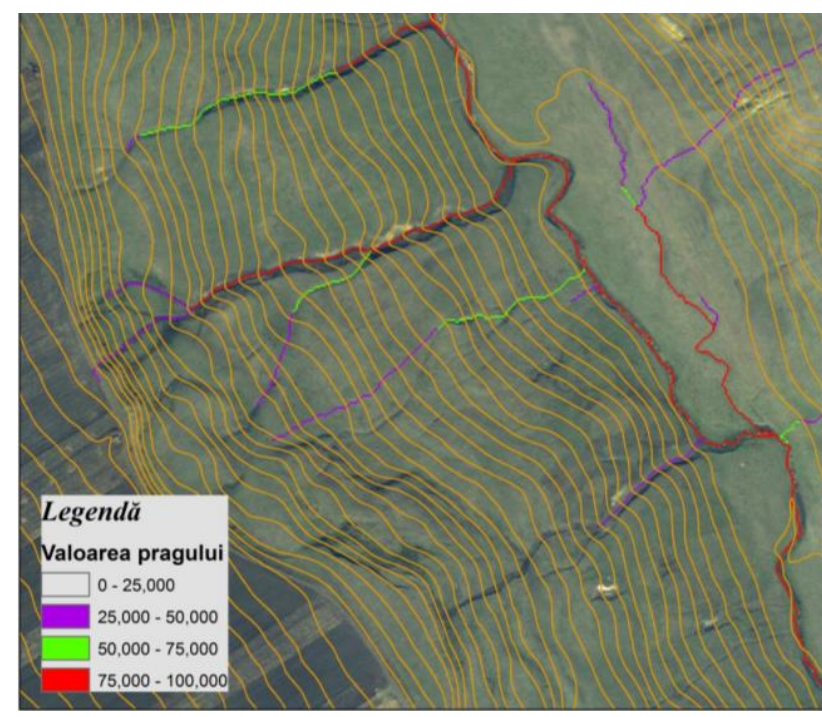

Figure 4. Accumulation raster simbolized upon different treshhold values for pixels

Taking into account these effects it is very important to establish the classification threshold value. A default value derived from the clasification method used is trying to cover general needs of classification. Refining the threshold value is, in our case, a necessity for obtaining some water courses compatible with the referenced topographic plan precision.To establish the best classification threshold there may be performed a comparison with the water's representation on cartographic data sours on equivalent scales, or on older topographic maps. These offer information regarding the waters position and density.Finding the optimum classification threshold value can be performed both by successively trials until obtaining the satisfying hydrographic value or by using the raster symbolization capabilities according to the pixels values.

\section{UPDATING THE HYDROLOGY USING COMBINED DATA SOURCE BLOCK}

If the LiDAR data are not homogeneous for the entire area of interest, in order to complete and rectify the data there are several initial data source that could be used. This method was selected to update the Hydrography theme of the Romanian topographic digital referenced plan. The following data sources are used:

- The vectors that represent la last version of the not updated running water track, as a data model respecting the INSPIRE requirements. This Feature Class (FC) is used as a base layer, thus conserving the textual part of the data. The version is updated by analyzing all the existing data source, both graphic and textual.

- The ortho photo plan in a mozaic shape with a $50 \mathrm{~cm}$ GSD, that includes, according to the location, the most recent available versions. The 2014 version is available for the North-East and East side of the country.

- The ortho photo plan in an infra-red spectrum (IR) represents a data source useful in the process of updating, especially for identifying the water courses and moisture excess in the areas with vegetation.

- Topographic plans scale 1:5.000 made by I.G.F.C.O.T that represent the topographic referenced plan for 
Romania before TopRo5. This source offers information on location and flowing water track as well as the density of the Hydrographic Network.

- The map for Romania scale 1:25.000 made by the Military Topographic Division representing the cartographic referenced document for small scales.

- Contour lines with a $1 \mathrm{~m}$ equidistance generated from LiDAR data. They are used as a support to confirm/infirm the existence and the track of the water flows. The cover over $20 \%$ of the Romanian surface. It also represents a nowadays data source, respectively 2010-2012.

- The track of some automatic generated rivers, by probabilistic algorithms, from LiDAR data. These are used for clarification of some water courses and some more elaborated land improvement. Being Hydrological model they are also useful to represent floods that are not visible otherwise, especially on flat areas.

- The Atlas for Romanian Drying up waters, 1974 edition, made by IGFCOT, together with The Institute for Meteorology and Hydrology. It helps to fulfill some values for the waters attributes, as well as to identify the track or to understand the context of a certain water within the hydrographic basin.

- The Atlas for Romanian Waters Cadastre, 1992 Edition, made by the Environment Ministry and having 130 maps scale 1:100.000 attached. It has the same role as the Atlas mentioned above, but it contains more updated, more detailed and more complete data from the waters cadastre point of view.

- Other topographic details of the referenced plan that interact with the Hydrographic Theme, such as channels or bridges.

- $\quad$ Satellite imaginary

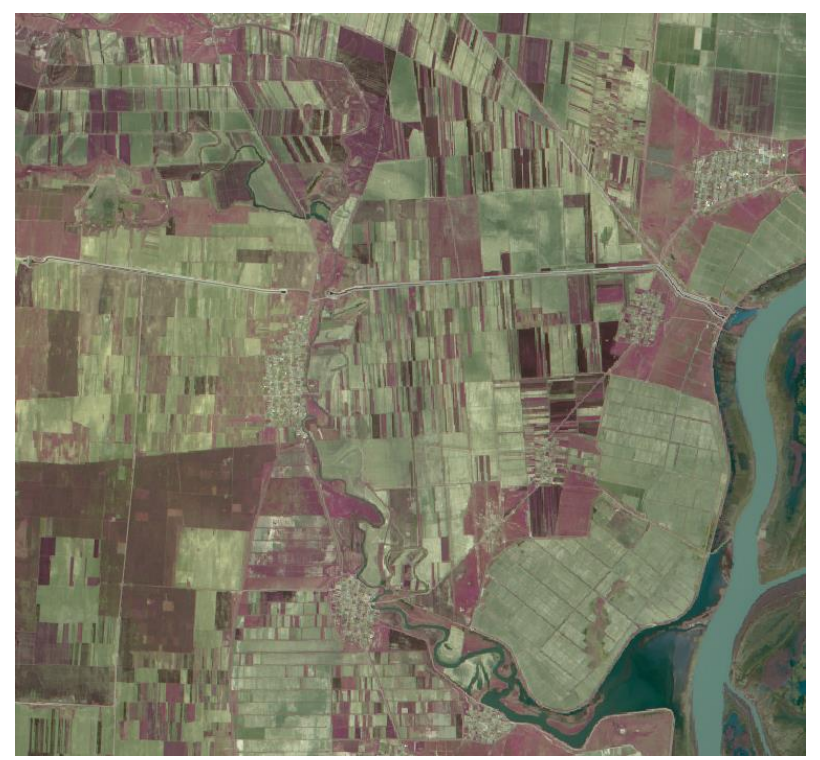

Figure 5. Santinel 2 Satelite imaginery

Band combinations for different satellite imaginary offers valuable information, in close relation to an apropriate scale.
Including all the data source in the process of updating we ensure a coherent procedure both for geometry and attribute values analizing every element independently.

Data management is carried out by using data bases, structured in such manner that coincide with the main steps of the project. All the process is taking place in a GIS environment because it offers an easy possibility to visualize and edit the attributes.

The method includes not only the 2D photo interpretation on the ortho photo plan but also a simultaneous analysis of all the data sources presented, offering additional information and the possibility to obtain more accurate the planimetric position and attributes of the water courses and lakes.

Furthermore, because it is not an automatic process it gives the possibility to the operator to intervene with its experience in solving some contradicting situations between the data sources. In these situations, the criteria of updating and accuracy are maintained in order to build a valid data base and relevant textual information is collected from all the data source available.

The fourth dimension, time is ensured both geometric and textual by stocking the "historic" type of information with the specification of the moment.

The entire process will have the $2 \mathrm{D}$ hydrographic actualized vectors as a result. Later they could be transformed into $3 \mathrm{D}$ vectors with the help of LiDAR data. During this transformation no information will be lost.

The expected results will offer the possibility of achieving statistics on large areas, such as hydrographic watershed, or any other purposes where the topographic data are needed.

\section{CONCLUSION}

The interdependent relationship between the hydrographic and relief layers lead to the need of an updating process where the two layers have to be envolved

Using more data sources means less geometric errors for hydrography and metadata by choosing the most probable version.

The automatic generation of the water courses obtained from LiDAR data represents a topical issue that may improve the process of obtaining and/or update a derived raster product that can be used as a data source for further versions or even a polyline type data representing the $2 \mathrm{D}$ hydrographic network.

Although it offers a good productivity compared to the classic vectorization, the automatic process of water courses generation cannot substitute the photo interpretation experience of a trained operator. The obtained results need its interpretation, especially in flat areas.

To apply successfully the mentioned method, and to obtain derived products, such as contour lines, digital terrain model or digital surface model, there must be given a great importance to the input data. The points density, the spatial precision as well as the temporal one are some of the items that may confirm the quality of the data. Also, the data processing (filtration, classification, generalization, etc.) must insure as much as possible the conservation of the primary data.

Thus, the implementation of some national standards for the quality of the geospatial data may insure the needed accuracy and homogeneity for their use as primary sources of information with lots of applications. 


\section{REFERENCES}

Greenlee, D. D. 1987. Raster and Vector Processing for Scanned Linework. Photogrammetric Engineering and Remote Sensing 53 (10): 1383-1387.

Jenson S. K. and J. O. Domingue. 1988. Extracting Topographic Structure from Digital Elevation Data for Geographic Information System Analysis. Photogrammetric Engineering and Remote Sensing 54 (11): 1593-1600.

Heidemann,H.K. Lidar Base Specification, Chapter 4 of Section B, U.S.Geological Survez Standards, Version 1.2, November 2014

National Oceanic and Atmospheric Administration (NOAA) Coastal Services Center. 2012. "Lidar 101: An Introduction to Lidar Technology, Data, and Applications.” Revised. Charleston, SC: NOAA Coastal Services Center.

ArcGIS Resources, www.geonet.esri.com/community, accessed March 2016 